\title{
GENERATION AND AMPLIFICATION OF ULTRASHORT UV PULSES VIA PARAMETRIC FOUR-WAVE INTERACTIONS IN TRANSPARENT SOLID-STATE MEDIA
}

\author{
A. Dubietis, J. Darginavičius, G. Tamošauskas, G. Valiulis, and A. Piskarskas \\ Department of Quantum Electronics, Vilnius University, Sauletekio 9 bldg. 3, LT-10222 Vilnius, Lithuania \\ E-mail: audrius.dubietis@ff.vu.lt
}

Received 15 June 2009; accepted 18 December 2009

\begin{abstract}
We report on efficient generation of the ultrashort ultraviolet light pulses by means of phase-matched four-wave optical parametric amplification and four-wave difference frequency mixing in UV-transparent solids with cubic nonlinearity. Broadband four-wave optical parametric amplification around $330 \mathrm{~nm}$ is investigated theoretically and demonstrated experimentally in optically isotropic materials, such as fused silica and $\mathrm{CaF}_{2}$ crystals. The results suggest that powerful broadband ultraviolet pulses as short as $7.5 \mathrm{fs}$ (in the transform limit) could be amplified in the field of milijoule 1-ps visible pulses. Efficient generation of 1-ps pulses at the $3 \mathrm{rd}(351 \mathrm{~nm}), 5$ th $(211 \mathrm{~nm})$, and, in particular, 6th $(176 \mathrm{~nm})$ harmonics of the Nd:glass laser is experimentally demonstrated through non-collinearly phase-matched four-wave difference-frequency mixing in fused silica, $\mathrm{CaF}_{2}$ and $\mathrm{MgF}_{2}$ crystals.
\end{abstract}

Keywords: Four-wave mixing, optical parametric amplification

PACS: 42.65.Jx, 42.25.Tg, 42.65.Yj, 42.65.Hw

\section{Introduction}

Generation and applications of the ultrashort light pulses in the ultraviolet attract much scientific and technological interest since the inception of nonlinear optics. Femtosecond ultraviolet laser pulses are on demand for many spectroscopic applications studying ultrafast processes in photochemistry and photobiology [1]. Many challenging applications in material processing, such as laser processing via surface ablation [2], microprinting of biomaterials [3], long-period fibre grating fabrication [4], in-depth modification of transparent solids [5], laser mirostructuring [6], to mention a few, require femtosecond laser radiation with energy of tens of microjoules. Among the variety of nonlinear optical techniques to generate ultrashort light pulses in the ultraviolet, four-photon interactions in transparent isotropic media attract a steadily growing interest. In particular, the four-wave mixing in noble gasses and air allows a direct generation and/or amplification of the ultrashort ultraviolet light pulses and has become a convenient technique to produce microjoule ultraviolet pulses with duration of a few tens of femtoseconds [7-13]. Recent advances in generation of few-cycle ul- traviolet pulses in gasses now challenge extreme pulse durations of $\sim 1$ fs $[14,15]$.

However, experimental studies of the four-wave interactions in transparent solids were limited mostly to generation and amplification of the ultrashort light pulses in the visible and infrared spectral range, using tightly focused laser beams [16-20], with quite a few studies devoted to ultraviolet pulse generation. In particular, third-harmonic generation was experimentally demonstrated, applying different interaction schemes, with [21] or without [22] assistance of the second-order nonlinearity in UV-transparent birefringent crystals as well as difference-frequency generation [24] and direct frequency tripling [25] in liquids. Despite the fact that condensed media possess much larger third-order susceptibility as compared to gasses, severe limitations are imposed by high material dispersion and high intensity, whose ultimate manifestation - laser beam and pulse self-action effects - eventually lead to the optical breakdown of the nonlinear medium. Hence efficient fourwave interactions in solids and liquids are difficult to achieve, and the energy of the generated pulses is limited to several microjoules at the best.

Recently, a new route in practical implementation of efficient four-wave optical parametric amplification 
in condensed bulk media was suggested making use of cylindrical focusing geometry, which allows one to fulfil the noncollinear phase-matching condition without reducing the interaction length. It is important to note that in this configuration, catastrophic selffocusing and break-up of high-intensity elliptical pump beam is quenched by the injection of a weak seed signal. As a result, the nonlinearly coupled pump, signal, and idler beams simultaneously reshape into stable 1-dimensional spatial solitons, where noncollinearly propagating signal and idler beams provide a damping mechanism that efficiently suppresses spatial and temporal instabilities [26,27]. Under these settings, the four-wave parametric amplification could be performed with high energy (millijoule) pump pulses, at the same time keeping the laser beam intensity reasonably below the optical damage threshold of the nonlinear medium. Taking an advantage of the specific shape of the phase matching curve, the four-wave optical parametric amplifier might provide a broad amplification bandwidth in the near infrared [28]. More recently, feasibility of this technique has been demonstrated in the ultraviolet spectral range for broadband four-wave parametric amplification [29] and high energy third harmonic generation of the Nd:glass laser by means of differencefrequency generation [30].

In this paper we overview the results of generation of high energy ultrashort light pulses in the UVDUV-VUV spectral range by means of four-wave optical parametric amplification and four-wave differencefrequency mixing in UV-transparent solid state bulk materials, such as fused silica, $\mathrm{CaF}_{2}$, and $\mathrm{MgF}_{2}$.

\section{Four-wave optical parametric amplification}

\subsection{Analytical model}

Four-wave optical parametric amplification in transparent isotropic media relies on the third-order nonlinear polarization that couples signal, idler, and two pump waves. A simple theory describing four-photon parametric interactions in condensed media along with phase-matching considerations is presented in [31]. In this section we extend this theory and develop an analytical model of the four-wave parametric amplification in a transparent isotropic bulk medium with account for the self- and cross-phase modulation experienced by the intense pump and weak signal and idler waves, respectively, in the plane and monochromatic wave approximation. The use of this approximation was justified according to the geometrical settings of the experiment, where the interacting beams in the phasematching plane could be regarded as plane waves. The complex amplitude equations describing evolution of coupled waves along the propagation direction $z$ have the form

$$
\begin{aligned}
\frac{\partial A_{1}}{\partial z}= & \sigma_{1} A_{3}^{2} A_{2}^{*} \mathrm{e}^{-\mathrm{i} \Delta k z}- \\
& \mathrm{i} \sigma_{1}\left(\left|A_{1}\right|^{2}+2\left|A_{2}\right|^{2}+2\left|A_{3}\right|^{3}\right) A_{1}, \\
\frac{\partial A_{2}}{\partial z}= & \sigma_{2} A_{3}^{2} A_{1}^{*} \mathrm{e}^{-\mathrm{i} \Delta k z}- \\
& \mathrm{i} \sigma_{2}\left(2\left|A_{1}\right|^{2}+\left|A_{2}\right|^{2}+2\left|A_{3}\right|^{2}\right) A_{2}, \\
\frac{\partial A_{3}}{\partial z}= & -2 \sigma_{3} A_{1} A_{2} A_{3}^{*} \mathrm{e}^{\mathrm{i} \Delta k z}- \\
& \mathrm{i} \sigma_{3}\left(2\left|A_{1}\right|^{2}+2\left|A_{2}\right|^{2}+\left|A_{3}\right|^{2}\right) A_{3},
\end{aligned}
$$

where $A_{j}(j=1,2,3)$ are the complex amplitudes of the signal, idler, and pump waves, respectively. We assume that the pump wave is composed of two collinear wave vectors $\mathbf{k}_{\mathbf{3}}$, that is, the pump wave is represented as a single intense laser beam, as schematically shown in the inset of Fig. 1. $\Delta k=\mathbf{k l}_{\mathbf{z}}$ is the longitudinal phase mismatch, where $\mathbf{I}_{\mathbf{z}}$ is the unit vector along $z$ axis, $\mathbf{k}=2 \mathbf{k}_{\mathbf{3}}-\mathbf{k}_{\mathbf{1}}-\mathbf{k}_{\mathbf{2}},\left|k_{j}\right|=\omega_{j} n_{0}\left(\omega_{j}\right) / c$, and $\sigma_{j}$ are the nonlinear wave coupling coefficients expressed as

$$
\sigma_{j}=\frac{3}{8} \frac{\omega_{j}}{n_{0}\left(\omega_{j}\right) c} \chi_{\mathrm{eff}}^{(3)}
$$

where $n_{0}\left(\omega_{j}\right)$ is the linear refractive index, $\omega_{j}$ is the frequency, and $\chi_{\text {eff }}^{(3)}$ is the third-order susceptibility related to the nonlinear refractive index $n_{2}$,

$$
n_{2}=\frac{3 \chi_{\text {eff }}^{(3)}}{4 n_{0}^{2}\left(\omega_{3}\right) c \epsilon_{0}} .
$$

The first terms on the right-hand side of Eqs. (1)-(3) describe the four-wave parametric amplification, while the second terms describe the self-phase modulation (SPM) of an intense pump wave and the cross-phase modulation (XPM) experienced by weak signal and idler waves. In the undepleted pump approximation $\left(\left|A_{3}\right| \gg\left|A_{2}\right|,\left|A_{1}\right|\right)$ Eqs. (1)-(3) reduce to

$$
\begin{aligned}
& \frac{\partial A_{1}}{\partial z}=\alpha_{1} \mathrm{e}^{\mathrm{i}\left(\beta_{3}-\Delta k\right) z} A_{2}^{*}+\mathrm{i} \beta_{1} A_{1}, \\
& \frac{\partial A_{2}}{\partial z}=\alpha_{2} \mathrm{e}^{\mathrm{i}\left(\beta_{3}-\Delta k\right) z} A_{1}^{*}+\mathrm{i} \beta_{2} A_{2} .
\end{aligned}
$$


Here we have introduced parameters $\alpha_{j}=\sigma_{j} A_{30}^{2}$ and $\beta_{j}=-2 \sigma_{j} A_{30}^{2}=-2 \alpha_{j}(j=1,2,3)$, where $A_{30}$ is the input amplitude of the pump wave. The coefficients $\alpha_{j}$ and $\beta_{j}$ describe relevant physical effects: the fourwave parametric amplification and the self- and crossphase modulation, respectively. The latter two are responsible for occurrence of the phase mismatch $\left(\beta_{3} \neq\right.$ $0)$ and the nonlinear phase shift $\left(\beta_{1,2} \neq 0\right)$. With boundary conditions for the input wave amplitudes $A_{10} \neq 0$ and $A_{20}=0$, i. e. only the seed signal wave present at the amplifier input, the solution of Eqs. (6) and (7) for the amplified signal amplitude has a form

$$
A_{1}(z)=A_{10}\left[\cosh (\Gamma z)+\frac{\mathrm{i}\left(\beta_{1}-\kappa\right)}{\Gamma} \sinh (\Gamma z)\right] \mathrm{e}^{\mathrm{i} \kappa z}
$$

where $\kappa=\left(\beta_{1}-\beta_{2}+\beta_{3}-\Delta k\right) / 2$ is the nonlinear wave number shift and $\Gamma$ is the gain increment expressed as

$$
\Gamma=\sqrt{\left[\alpha_{1} \alpha_{2}-\beta_{1} \beta_{2}+\beta_{1}\left(\beta_{3}-\Delta k\right)\right]-\kappa^{2}} .
$$

Note that parameters $\alpha_{j}, \beta_{j}, \kappa$, and $\Delta k$ are functions of frequency. The four-wave parametric process has a gain only if $\Gamma$ is real. The amplified signal amplitude is therefore a function not only of the (linear) phase mismatch $\Delta k$, but also crucially depends on the nonlinear effects imposed by intense pump wave.

\subsection{Analysis of the parametric gain profiles}

Next we consider the phase matching for the fourwave parametric amplification in fused silica. The phase matching angle versus wavelength is plotted in Fig. 1. The character of the phase matching curve indicates only a small change in the phase matching angle $\theta_{\mathrm{pm}}$ for signal wavelengths around $\sim 330 \mathrm{~nm}$, where the broadband amplification could be expected.

In what follows, using the developed analytical model, we study the four-wave optical parametric amplification in this wavelength range. Since our analytical model is for monochromatic waves, we reconstruct the four-wave parametric gain profile by varying the signal wavelength and keeping $\theta_{\mathrm{pm}}$ fixed. In the calculations we take $I_{3}=160 \mathrm{GW} / \mathrm{cm}^{2}, n_{2}=$ $2.2 \cdot 10^{-16} \mathrm{~cm}^{2} / \mathrm{W}, z=5 \mathrm{~mm}$. The dashed curve in Fig. 2(a) plots the phase mismatch $\Delta k$ versus signal wavelength as if a perfect phase matching $\left(\Delta k_{0}=0\right)$ is set for the signal wavelength $\lambda_{1}=330 \mathrm{~nm}$. The solid curve indicates the phase mismatch, $\Delta k_{\mathrm{n}_{2}}$, with account for nonlinear terms, i. e. $\mathbf{k}_{\mathrm{n}_{2}}=\mathbf{2} \mathbf{k}_{\mathbf{3}}^{\left(\mathrm{n}_{2}\right)}-\mathbf{k}_{\mathbf{2}}^{\left(\mathrm{n}_{2}\right)}-\mathbf{k}_{\mathbf{1}}^{\left(\mathrm{n}_{2}\right)}$ associated with the modification of the phase matching

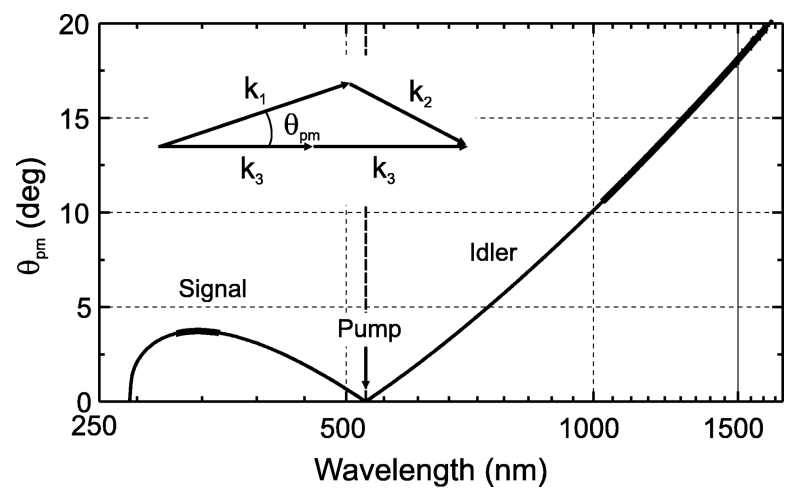

Fig. 1. Phase-matching curve for the four-wave parametric amplification pumped at $\lambda_{3}=527 \mathrm{~nm}$ in fused silica. Wavelength range for expected broadband amplification of the signal wave and the corresponding idler wavelength range are highlighted by the bold curves. Inset shows the wave vector diagram.

condition due to SPM and XPM via the nonlinear refractive index:

$$
\begin{aligned}
& \left|k_{1}^{\left(\mathrm{n}_{2}\right)}\right|=\frac{\omega_{1}}{c}\left[n_{0}\left(\omega_{1}\right)+2 n_{2} I_{3}\right], \\
& \left|k_{2}^{\left(\mathrm{n}_{2}\right)}\right|=\frac{\omega_{2}}{c}\left[n_{0}\left(\omega_{2}\right)+2 n_{2} I_{3}\right], \\
& \left|k_{3}^{\left(\mathrm{n}_{2}\right)}\right|=\frac{\omega_{3}}{c}\left[n_{0}\left(\omega_{3}\right)+n_{2} I_{3}\right],
\end{aligned}
$$

where the pump wave intensity is defined as $I_{3}=$ $c n_{0}\left(\omega_{3}\right) \epsilon_{0}\left|A_{30}\right|^{2} / 2$. The dotted curve illustrates the imaginary part of the gain increment, $\operatorname{Im} \Gamma$, expressed in terms of phase mismatch. Figures $2(b, c)$ show the change in quantities of $\Delta k, \Delta k_{\mathrm{n} 2}$, and $\operatorname{Im} \Gamma$ with imposing some initial phase mismatch $\Delta k_{0}$ for the central signal wavelength. The impact of the phase mismatch on the signal amplitude versus signal wavelength is illustrated in Fig. 2(d) by plotting the argument $\Gamma z$ for the three $\Delta k_{0}$ cases discussed above, i. e. $\Delta k_{0}=0, \Delta k_{0}=$ $1.5 \mathrm{~cm}^{-1}$, and $\Delta k_{0}=3 \mathrm{~cm}^{-1}$.

The SPM and XPM induced nonlinear phase shift results in some additional phase mismatch for the signal wavelengths at the vicinity of the central frequency, where $\Gamma z$ drops to zero. The perfect phase matching $\Delta k=0$ therefore occurs for the shifted frequencies, as evident from Fig. 2(a), and thus results in splitting of the parametric gain profile. By compensating the nonlinear phase shift with introducing a positive $\Delta k_{0}$ (for instance, by slight adjustment of the crossing angle between the pump and signal beams) the gain profile takes a homogeneous shape, with two distinct frequency-shifted peaks.

Figure 3 plots the four-wave parametric gain $G(\lambda)$ versus signal wavelength calculated by use of Eqs. (8) 
(a)

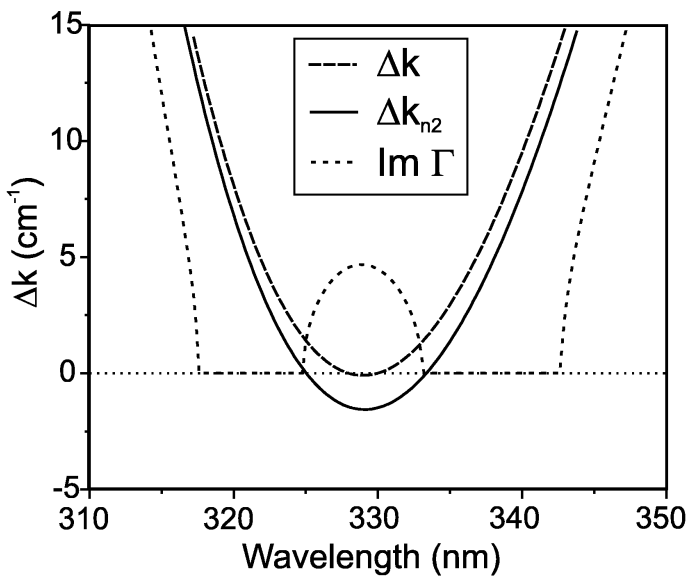

(c)

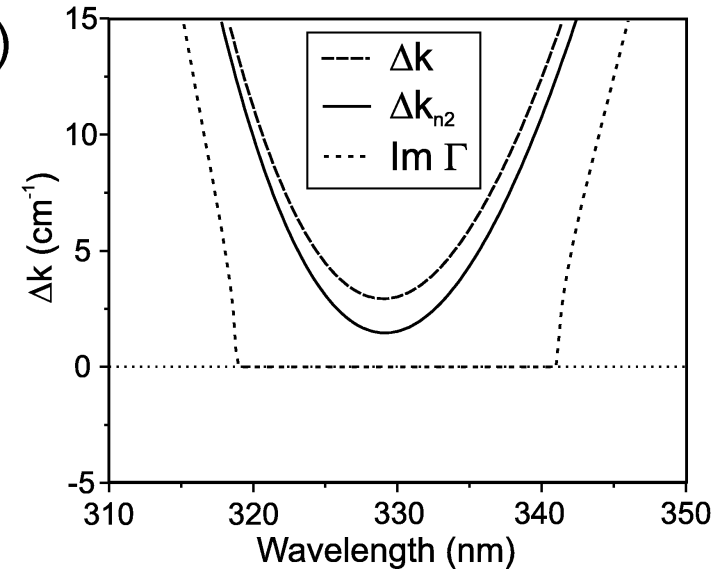

(b)

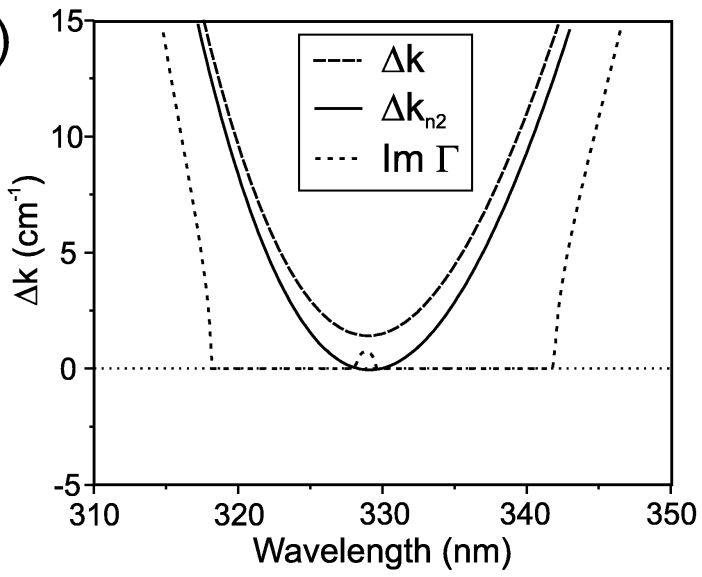

(d)

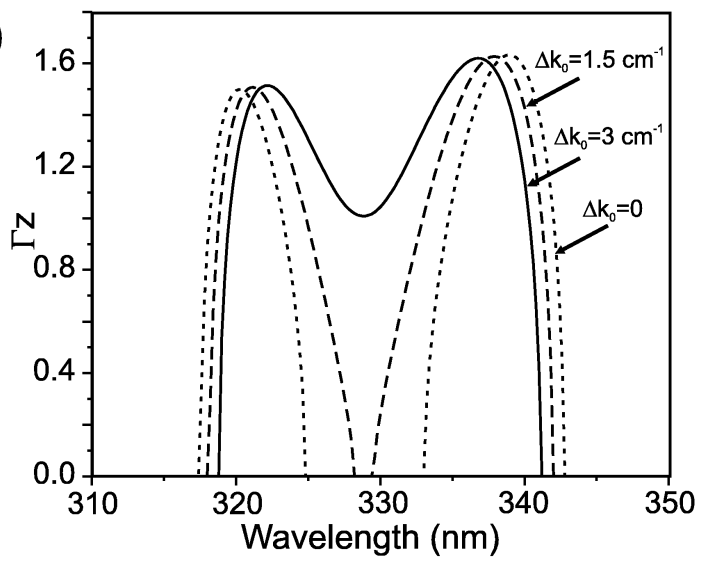

Fig. 2. Phase mismatch $\Delta k, \Delta k_{\mathrm{n} 2}$, and $\operatorname{Im} \Gamma$ in fused silica versus signal wavelength: (a) $\Delta k_{0}=0$, (b) $\Delta k_{0}=1.5 \mathrm{~cm}^{-1}$, (c) $\Delta k_{0}=3 \mathrm{~cm}^{-1}$. Here $\Delta k_{0}$ denotes the phase mismatch for the central signal wavelength of $330 \mathrm{~nm}$. (d) Argument $\Gamma z$ versus signal wavelength calculated for three different values of $\Delta k_{0}$ with $I_{3}=160 \mathrm{GW} / \mathrm{cm}^{2}$. See text for details.

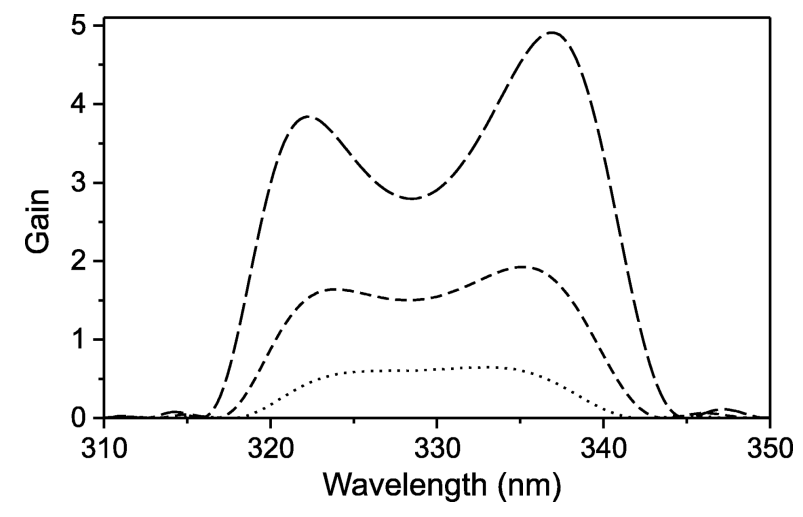

Fig. 3. Calculated four-wave parametric gain profiles in fused silica for three different pump intensities with $\Delta k_{0}=3 \mathrm{~cm}^{-1}$ : dotted curve for $I_{3}=80 \mathrm{GW} / \mathrm{cm}^{2}$, short-dash curve for $I_{3}=$ $120 \mathrm{GW} / \mathrm{cm}^{2}$, long-dash curve for $I_{3}=160 \mathrm{GW} / \mathrm{cm}^{2}$.

and (9) with $\Delta k_{0}=3 \mathrm{~cm}^{-1}$ for three different pump intensities. Here gain is defined as

$$
G(\lambda)=\left|\frac{A(z, \lambda)}{A_{10}}\right|^{2}-1
$$

The plot suggests that the four-wave parametric gain bandwidth broadening is resulting from the SPM and XPM, whose contribution increases with increasing pump intensity.

\subsection{Experimental results}

The theoretical findings described in the preceding section were verified experimentally using an amplified Nd:glass laser system (Twinkle, Light Conversion Ltd.), which delivered frequency-doubled 1-ps pulses with $3 \mathrm{~mJ}$ energy at $527 \mathrm{~nm}$ and $10 \mathrm{~Hz}$ repetition rate. The laser output was split into two parts. The smaller portion of the laser radiation $(1 \mathrm{~mJ})$ was made variable in energy by means of the attenuator $(\lambda / 2$ plate and thin film polarizer) and served as a pump for the four-wave optical parametric amplifier (FWOPA). The larger portion of the laser radiation $(2 \mathrm{~mJ})$ was used to pump the commercial three-wave optical parametric generator/amplifier system (Topas, Light Conversion Ltd.), which after a supplementary frequency upconversion stage (e.g. sum-frequency mixing with a fresh $527 \mathrm{~nm}$ radiation) delivered tunable ultraviolet 
pulses in the range of 310-365 $\mathrm{nm}$. These pulses further served as a seed signal for the FWOPA. The temporal delay between co-polarized pump and seed signal pulses was adjusted using a motorized delay line. The pump and seed signal beams were focused with cylindrical lenses $\left(f_{y}=+750 \mathrm{~mm}, f_{x}=\infty\right.$ and $f_{y}=$ $+500 \mathrm{~mm}, f_{x}=\infty$, respectively), so as to ensure mode matching at the input face of the nonlinear medium. Cylindrical focusing yielded respective FWHM dimensions of the pump and seed signal beams of $4.7 \mathrm{~mm} \times$ $55 \mu \mathrm{m}$ and $3 \mathrm{~mm} \times 50 \mu \mathrm{m}$. Geometrically, the beams were arranged as follows: while the cylindrical focusing was performed in the vertical plane, the beams were crossed in the horizontal plane at the phase matching angle $\theta_{\mathrm{pm}}=3.7^{\circ}$, thereby ensuring a good spatial overlap along the entire length of the nonlinear medium. As an amplifying medium we have chosen a 5-mm-long UV-grade (type KU-1) fused silica sample.

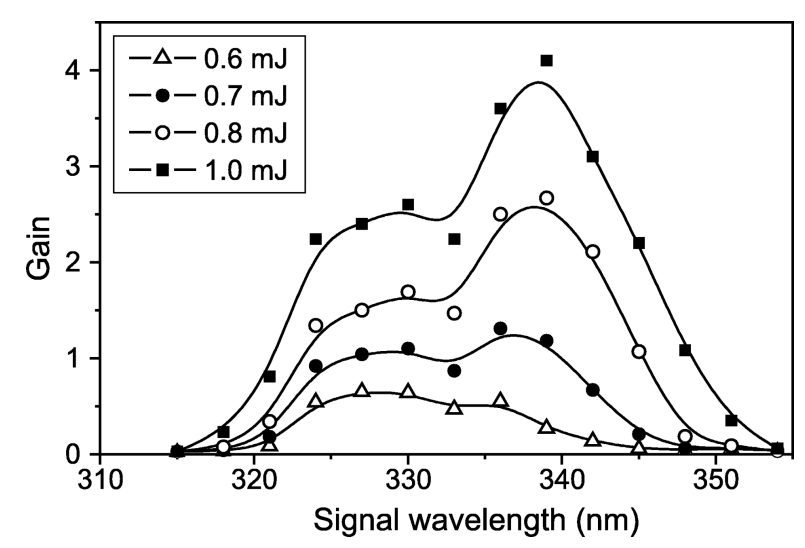

Fig. 4. Experimentally measured four-wave parametric gain profiles versus pump energy in 5-mm fused silica sample.

FWOPA was seeded by a narrow-band (FWHM width $\sim 1 \mathrm{~nm}$ ) signal with an input energy $E_{10}=10 \mu \mathrm{J}$. The wavelength of the seed signal was tuned from 315 to $355 \mathrm{~nm}$ in $3 \mathrm{~nm}$ step, so as to scan precisely the gain profile of the FWOPA, while the beam crossing angle was kept fixed. Figure 4 represents the experimentally obtained parametric gain profiles, measured at different pump energy. The experimental data is in fair agreement with the calculations, although the range of experimental intensity values $\left(450-750 \mathrm{GW} / \mathrm{cm}^{2}\right)$ is higher than that used in the calculations. This difference could be in part attributed to the spatial and temporal dynamics of the pump beam during its propagation within the nonlinear medium, and this was not taken into account in the analytical model.

With the highest applicable pump energy $E_{3}=$ $1 \mathrm{~mJ}$ (limited by the optical breakdown of fused silica, whose threshold is $F_{\text {th }}=1.3 \mathrm{~J} / \mathrm{cm}^{2}$ for 1-ps, 526-nm pulses [32]), the FWHM gain bandwidth broadens up to $21 \mathrm{~nm}$, which is wide enough to support the fourwave optical parametric amplification of light pulses as short as $7.5 \mathrm{fs}$ (in the transform limit). We also note that with $E_{3}=1 \mathrm{~mJ}$ the highest amplified signal energy of $55 \mu \mathrm{J}$ was measured with almost $4 \%$ pump-tosignal energy conversion efficiency in the gain saturation regime. Similar results had been obtained by replacing fused silica with $l=6 \mathrm{~mm} \mathrm{CaF}_{2}$ sample and reported elsewhere [29].

\section{Generation of the ultraviolet light pulses via four-wave difference-frequency mixing}

The most straightforward way to convert the radiation emitted by solid-state lasers into the ultraviolet spectral range is the third-harmonic generation. Formally, the presence of the cubic nonlinearity in all transparent materials allows direct conversion of three laser photons at frequency $\omega$ into a single one at $3 \omega$ $(3 \omega=\omega+\omega+\omega)$. However, in a medium with normal group velocity dispersion, the nonresonant thirdharmonic generation process could not be exactly phase matched, since the total length of three wave vectors at $\omega$ is always shorter than a length of a single wave vector of $3 \omega$. In general, third-harmonic generation in transparent media with normal group velocity dispersion could be phase matched: (i) with the help of non-perturbative effects, imposed by the intense laser beam propagation in gasses [7]; (ii) by choosing different polarization of the input waves in media without inversion symmetry [22]; (iii) by creation of transient refractive index grating with crossing laser beams [23]; (iv) via four-wave difference-frequency mixing $3 \omega=2 \omega+2 \omega-\omega$ in isotropic media [24]. The latter approach offers much more flexibility, since it allows the phase matching condition to be perfectly fulfilled in any transparent medium. And, more importantly, the four-wave difference-frequency mixing method could be easily extended to any other higher laser harmonic generation by choosing appropriate frequencies of the input radiation.

In what follows, we present the experimental results on efficient generation of 1-ps pulses at third, fifth, and sixth harmonics of the Nd:glass laser by phase matched four-wave difference-frequency mixing in transparent solids. A major achievement lies in the highly efficient generation of 1-ps light pulses in the deep and vacuum ultraviolet in bulk wide-bandgap solids. 


\subsection{Third-harmonic generation at $351 \mathrm{~nm}$ in fused silica}

In the experiment, the third harmonic of the Nd:glass laser at $351 \mathrm{~nm}$ was generated by mixing its fundamental $(1054 \mathrm{~nm})$ and second-harmonic $(527 \mathrm{~nm})$ pulses via difference frequency generation process, i. e. $3 \omega=$ $2 \omega+2 \omega-\omega$, in 3-mm-long UV-grade (type KU-1) fused silica sample. Intense fundamental and secondharmonic laser beams were cylindrically focused onto the input face of the fused silica sample and crossed at $\theta_{\mathrm{pm}}=11^{\circ}$. The FWHM diameter of both elliptical pump beams was measured as $5 \mathrm{~mm} \times 50 \mu \mathrm{m}$. The energy ratio of the two pump beams was adjusted as $E_{\omega}: E_{2 \omega}=1: 4$, according to Manley-Rowe relation in order to ensure optimal photon yield. The energy of the overall pump $E_{\mathrm{p}}=E_{\omega}+E_{2 \omega}$ was varied up to $1.55 \mathrm{~mJ}$, being limited by the optical damage of the fused silica sample.

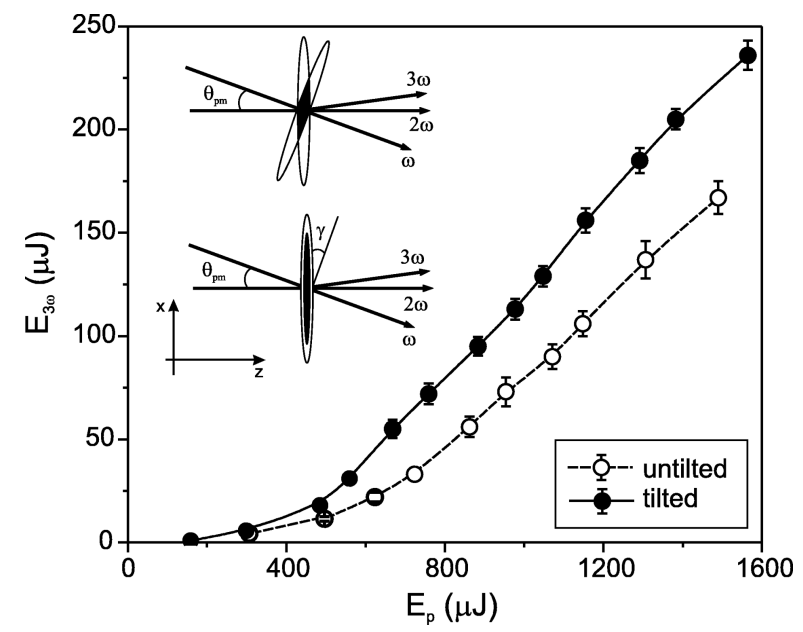

Fig. 5. Experimentally measured third-harmonic pulse energy $E_{3 \omega}$ versus pump energy with untilted (dashed curve and open circles) and tilted (solid curve and full circles) pulses. Inset shows pulse/beam overlap area in the phase matching plane with untilted (upper illustration) and tilted (lower illustration) pulses.

Geometrically, at large beam crossing angle $\left(\theta_{\mathrm{pm}}=\right.$ $11^{\circ}$ ), the physical beam/pulse overlap area within the nonlinear medium becomes greatly reduced, as illustrated in the upper picture of the inset of Fig. 5. Note, that the cylindrically focused laser beam has a width of $5 \mathrm{~mm}$ FWHM along its long $(x)$ axis in the phase matching plane, whereas the physical length of 1-ps pulses inside the sample is just $\sim 0.2 \mathrm{~mm}$. Therefore noncollinearly propagating pump beams overlap only partially, yielding a narrow third-harmonic beam and, as a consequence, less efficient energy conversion. Therefore we have improved the overlap geometry by tilting the amplitude front of the fundamental-frequency pulse by $\gamma=8.6^{\circ}$, as schematically illustrated in the lower picture of the inset of Fig. 5. More technical details are provided in [30]. Figure 5 compares the results of thirdharmonic generation with untilted and tilted pulses by plotting third-harmonic pulse energy $E_{3 \omega}$ as a function of the pump energy. Indeed, pulse-front tilting of the fundamental-frequency pulse has led to an increase of the third-harmonic pulse energy by $25-30 \%$, with the highest value of $E_{3 \omega}=230 \mu \mathrm{J}$ as measured at maximum pump energy of $E_{\mathrm{p}}=1.55 \mathrm{~mJ}$. This result suggests the energy conversion efficiency as high as $15 \%$, which is the highest number to our knowledge achieved in the third-harmonic generation via pure cubic nonlinearity reported up to date.

\subsection{Fifth-harmonic generation at $211 \mathrm{~nm}$ in $\mathrm{CaF}_{2}$ and $M g F_{2}$}

The four-wave difference-frequency generation method in solids was further extended to even shorter wavelengths in the deep-UV, by demonstrating efficient fifth-harmonic generation in $\mathrm{CaF}_{2}$ and $\mathrm{MgF}_{2}$ samples. The phase matched fifth-harmonic generation was achieved by mixing third- and fourth-harmonic pulses of the Nd:glass laser (with wavelengths 351 and $264 \mathrm{~nm}$, respectively), producing the radiation at $211 \mathrm{~nm}$ through the difference-frequency mixing process $5 \omega=4 \omega+4 \omega-3 \omega$. The beam focusing and interaction geometry was essentially identical to those described in the previous sections, with the phase matching angles of $8.3^{\circ}$ and $7.2^{\circ}$ calculated for $\mathrm{CaF}_{2}$ and $\mathrm{MgF}_{2}$, respectively, and the input beam dimensions of $2.9 \mathrm{~mm} \times 30 \mu \mathrm{m}$ at the input face of the nonlinear medium. Since $\mathrm{MgF}_{2}$ crystal is birefringent, its optical axis was aligned at $0^{\circ}$ to normal incidence. The length of crystal samples was chosen as $l=4 \mathrm{~mm}$, as a compromise of the group velocity mismatch lengths between the fourth-harmonic and fifth-harmonic pulses of $3.2 \mathrm{~mm}$ in $\mathrm{CaF}_{2}$ and $4.1 \mathrm{~mm}$ in $\mathrm{MgF}_{2}$. The linear and nonlinear parameters of $\mathrm{CaF}_{2}$ and $\mathrm{MgF}_{2}$, relevant to the present experiment are listed in Table 1. Both crystals possess very similar threshold damage fluence as measured in the visible and near infrared [32], and considering that it scales inversely with frequency, the expected values are in the order of $\sim 0.5 \mathrm{~J} / \mathrm{cm}^{2}$ for 1-ps pulses at $264 \mathrm{~nm}$. The input energy ratio between the pump waves was set as $E_{4 \omega}: E_{3 \omega}=8: 3=2.6$, according to interacting photon energy. The overall pump energy was defined as $E_{\mathrm{p}}=E_{4 \omega}+E_{3 \omega}$ and was varied up to $\sim 0.5 \mathrm{~mJ}$. 
Table 1. Relevant linear and nonlinear properties of $\mathrm{CaF}_{2}$ and $\mathrm{MgF}_{2}$ crystals for fifthharmonic generation. $E_{\mathrm{g}}$ is the bandgap, $T$ is transmission range at $1 \mathrm{~cm}^{-1}$ level [34], $n_{2}$ is the nonlinear refractive index [34], $\theta_{\mathrm{pm}}$ is the phase matching angle calculated from the Sellemier equation [35], $L_{\mathrm{GVM}}$ is the group velocity mismatch length, calculated for

1 -ps pulses, $\beta_{264}$ is the two-photon absorption coefficient, measured in this work.

\begin{tabular}{lllllll}
\hline Crystal & $E_{\mathrm{g}, \mathrm{eV}}$ & $\mathrm{T}, \mu \mathrm{m}$ & $n_{2}, \mathrm{~cm}^{2} / \mathrm{W}$ & $\theta_{\mathrm{pm}}, \mathrm{deg}$ & $L_{\mathrm{GVM}}, \mathrm{mm}$ & $\beta_{264}, \mathrm{~cm} / \mathrm{W}$ \\
\hline $\mathrm{CaF}_{2}$ & 10.0 & $0.13-9.4$ & $4.2 \cdot 10^{-16}$ & 8.3 & 3.2 & $7.3 \cdot 10^{-12}$ \\
$\mathrm{MgF}_{2}$ & 11.8 & $0.13-7.4$ & $1.5 \cdot 10^{-16}$ & 7.2 & 4.1 & $1.9 \cdot 10^{-12}$ \\
\hline
\end{tabular}

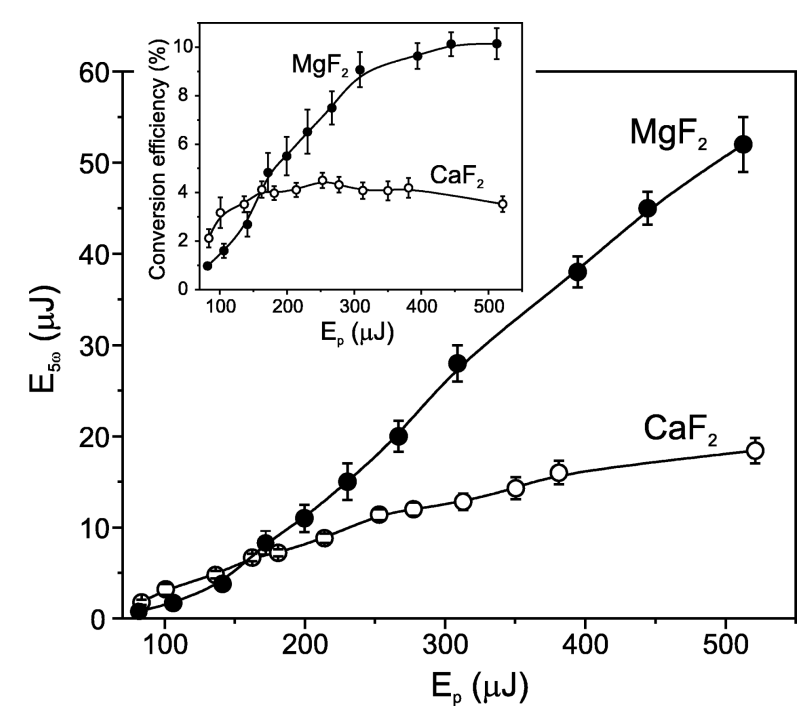

Fig. 6. Experimentally measured fifth-harmonic energy $E_{5 \omega}$ versus pump energy in $\mathrm{CaF}_{2}$ (open circles) and $\mathrm{MgF}_{2}$ (full circles) crystals. Inset shows the respective energy conversion efficiency of the fifthharmonic generation process.

Figure 6 summarizes the main results showing the fifth-harmonic pulse energy $E_{5 \omega}$ versus pump energy, measured in $\mathrm{CaF}_{2}$ and $\mathrm{MgF}_{2}$ crystals. The inset shows energy conversion efficiency calculated as $E_{5 \omega} /\left(E_{4 \omega}+\right.$ $\left.E_{3 \omega}\right)$ and demonstrates that the interaction regime in both media is driven into saturation. In $\mathrm{CaF}_{2}$, the highest energy conversion of $4.5 \%$ was measured at $E_{\mathrm{p}}=$ $250 \mu \mathrm{J}$, yielding the fifth-harmonic pulses with energy of $E_{5 \omega}=13 \mu \mathrm{J}$. Since the energy conversion saturates, further increase of the fifth-harmonic pulse energy, measured at higher pump energies is small $\left(E_{5 \omega}=\right.$ $18 \mu \mathrm{J}$ at $\left.E_{\mathrm{p}}=520 \mu \mathrm{J}\right)$. Significantly better results were achieved in $\mathrm{MgF}_{2}$ sample, where fifth-harmonic pulse energy as high as $E_{5 \omega}=52 \mu \mathrm{J}$ at $E_{\mathrm{p}}=500 \mu \mathrm{J}$ was measured, that equates to $\sim 10 \%$ energy conversion efficiency.

It is important to note that the shorter and the most intense pump wave, i.e. the fourth-harmonic pulse, is the subject to two-photon absorption. To verify this, we have accomplished the nonlinear transmission measurements in our samples with fourth-harmonic pulse alone, since exact values of the two-photon absorption coef-

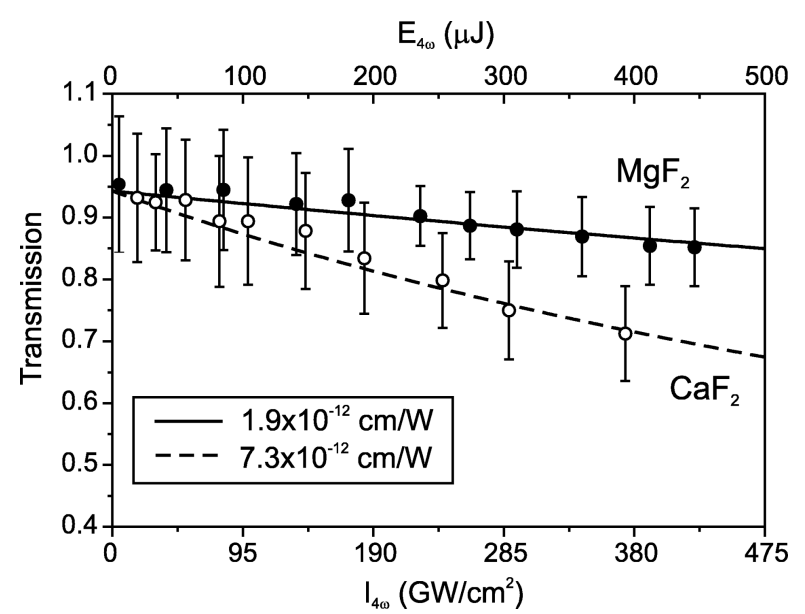

Fig. 7. Nonlinear transmission of 4-mm-long $\mathrm{CaF}_{2}$ (open circles) and $\mathrm{MgF}_{2}$ (full circles) samples versus fourth-harmonic pulse intensity. Dashed and solid curves show the best fit yielding two-photon absorption coefficients $\beta_{264}=7.3 \cdot 10^{-12} \mathrm{~cm} / \mathrm{W}$ and $\beta_{264}=$ $1.9 \cdot 10^{-12} \mathrm{~cm} / \mathrm{W}$, respectively.

ficients for $\mathrm{CaF}_{2}$ and $\mathrm{MgF}_{2}$ crystals are not provided in the literature. The nonlinear transmission measurement methodology and the two-photon absorption coefficient retrieval algorithm was essentially similar to that described in [33]. In the present experiment we have just ascertained that the dimensions of the input beam are not changed during the propagation in the sample. Indeed, the estimated Rayleigh range for the $30 \mu \mathrm{m}$ (FWHM) input beam is in the order of $\sim 15 \mathrm{~mm}$, and thus ensures that the intensity change associated with diffraction spread of the input beam were negligible during its propagation in the 4-mm-long sample. Figure 7 shows the results of the nonlinear transmission measurements in 4-mm-long $\mathrm{CaF}_{2}$ and $\mathrm{MgF}_{2}$ samples, along with the best-fit yielding two-photon absorption coefficients $\beta_{264}$ of $7.3 \cdot 10^{-12}$ and $1.9 \cdot 10^{-12} \mathrm{~cm} / \mathrm{W}$ for $\mathrm{CaF}_{2}$ and $\mathrm{MgF}_{2}$, respectively.

Taking into consideration the nonlinear parameters of both media, we can explain on simple grounds the marked differences in fifth-harmonic generation results obtained in $\mathrm{CaF}_{2}$ and $\mathrm{MgF}_{2}$ crystals, as depicted in Fig. 6. First of all, larger nonlinearity of $\mathrm{CaF}_{2}$ (that is in fact proportional to the nonlinear refractive index 
$n_{2}$ ) makes the fifth-harmonic generation process more efficient as compared to $\mathrm{MgF}_{2}$ in the input pump energy range of 80-150 $\mu \mathrm{J}$. Conversely, in the high pump energy limit, energy losses due to two-photon absorption of one of the pump waves (i.e. fourth-harmonic) in $\mathrm{CaF}_{2}$ are considerably larger that those in $\mathrm{MgF}_{2}$, so the further pump intensity increase has just very small effect on the fifth-harmonic pulse energy. On the other hand, the four-wave difference-frequency generation process in $\mathrm{MgF}_{2}$ saturates at higher pump intensity owing to its smaller nonlinearity, which combined with small two-photon absorption for the fourth-harmonic wave, allows much higher fifth-harmonic pulse energy to be achieved. It is worth mentioning that the nonlinear losses experienced by the fifth-harmonic pulse in $\mathrm{MgF}_{2}$ are also expected to be considerably lower than those in $\mathrm{CaF}_{2}$. And finally, we note that for the repetition rate of $10 \mathrm{~Hz}$ of our laser, permanent colour centre formation due to intense ultraviolet irradiation has not been observed in any of the used crystal samples.

\subsection{Generation of VUV radiation by four-wave difference-frequency mixing in $\mathrm{MgF}_{2}$}

Generation of the ultrashort light pulses in the vacuum ultraviolet in the solid state media is a formidable task because of high group velocity mismatch between the interacting pulses, high linear and nonlinear absorption, and phase matching constraints in the crystals with quadratic nonlinearity. To date, the shortest wavelengths generated via quadratic phase-matched interactions are reported in $\mathrm{LBO}(172.7 \mathrm{~nm})$ [36], $\mathrm{CsLiB}_{6} \mathrm{O}_{10}$ $(175 \mathrm{~nm})$ [37], $\mathrm{KB}_{5} \mathrm{O}_{8} \cdot 4 \mathrm{H}_{2} \mathrm{O}(166 \mathrm{~nm})$ [38] crystals. Typical generated pulse energy ranges from tens to hundreds of nanojoules in the femtosecond regime.

In this section we demonstrate high potential of wide-bandgap $\mathrm{MgF}_{2}$ crystal for the generation of VUV radiation by four-wave difference-frequency mixing. Specifically, we generate sixth-harmonic at $176 \mathrm{~nm}$ of the Nd:glass laser via mixing 1-ps fourth-harmonic and second-harmonic pulses in 2-mm-long $\mathrm{MgF}_{2}$ sample through four-wave difference-frequency mixing, $6 \omega=$ $4 \omega+4 \omega-2 \omega$, as schematically depicted in Fig. 8(a). The particular details of the experiment are as follows. The beam focusing conditions were identical as those in the fifth-harmonic generation case. The fourthharmonic and second-harmonic beams were crossed at the phase matching angle of $\theta_{\mathrm{pm}}=20^{\circ}$. In order to optimize the pulse/beam overlap area, the second-harmonic pulse-front was tilted by $\gamma=23^{\circ}$ using a diffraction grating with 600 lines $/ \mathrm{mm}$ at $43^{\circ}$ incidence and second diffraction order. The length of $\mathrm{MgF}_{2}$ crystal $(l=$
$2 \mathrm{~mm}$ ) was chosen to be close to the group velocity mismatch length calculated for 1-ps pulses $\left(L_{\mathrm{GVM}}=\right.$ $1.7 \mathrm{~mm}$ ). The energy of the sixth-harmonic pulse was measured using pyroelectric energy meter Ophir Nova II with pyroelectric detector head PE9-SH. The crystal and detector head were placed in a container with continuously refilled pure nitrogen atmosphere, so as to avoid strong absorption by the oxygen molecules. The energy ratio of the input beams was chosen as $E_{4 \omega}: E_{2 \omega}=8: 2=4$, and the overall pump energy was varied from 0.1 to $0.95 \mathrm{~mJ}$.

(a)

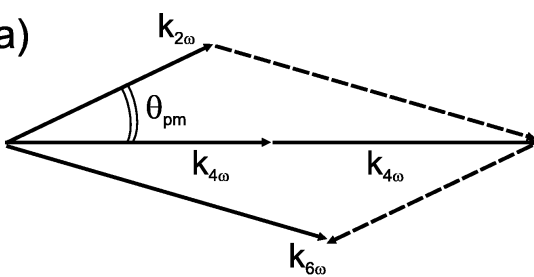

(b)

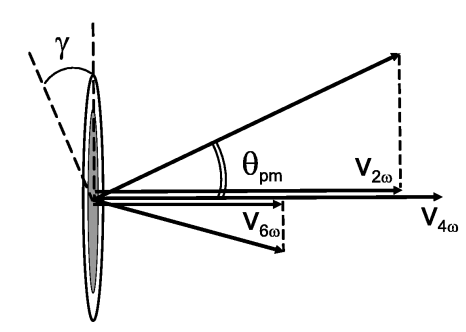

Fig. 8. (a) Wave vector diagram for the sixth-harmonic generation. Optical axis of $\mathrm{MgF}_{2}$ crystal is adjusted for normal incidence of the fourth-harmonic beam. (b) Schematic representation of the interacting beam/pulse overlap and pulse group velocities and their projections along the propagation axis.

The sixth-harmonic pulse energy $E_{6 \omega}$ and energy conversion efficiency versus pump energy is plotted in Fig. 9. The highest sixth-harmonic pulse energy of $E_{6 \omega}=10 \mu \mathrm{J}$ was measured at $E_{\mathrm{p}}=0.9 \mathrm{~mJ}$, that is the highest energy to our knowledge in the near VUV spectral range obtained with 1-ps pulses. The energy conversion curve exhibits a broad plateau for the input pump energy in the range of $0.5-0.9 \mathrm{~mJ}$, with almost constant energy conversion efficiency of $\sim 1.1 \%$, indicating that the conversion process is driven into saturation. The intensity profile of the sixth-harmonic beam was measured via imaging of the luminescence of the colour filter, placed into the beam path at the intermediate field, and is shown in the inset of Fig. 9. The beam becomes slightly deteriorated once the saturation regime is achieved, as a result of spatial intensity variation of the pump beams and subsequent modification of the phase matching condition whose mechanism is similar to that described in Section 2.2. It is important to note that even at high UV and VUV irradiation flu- 


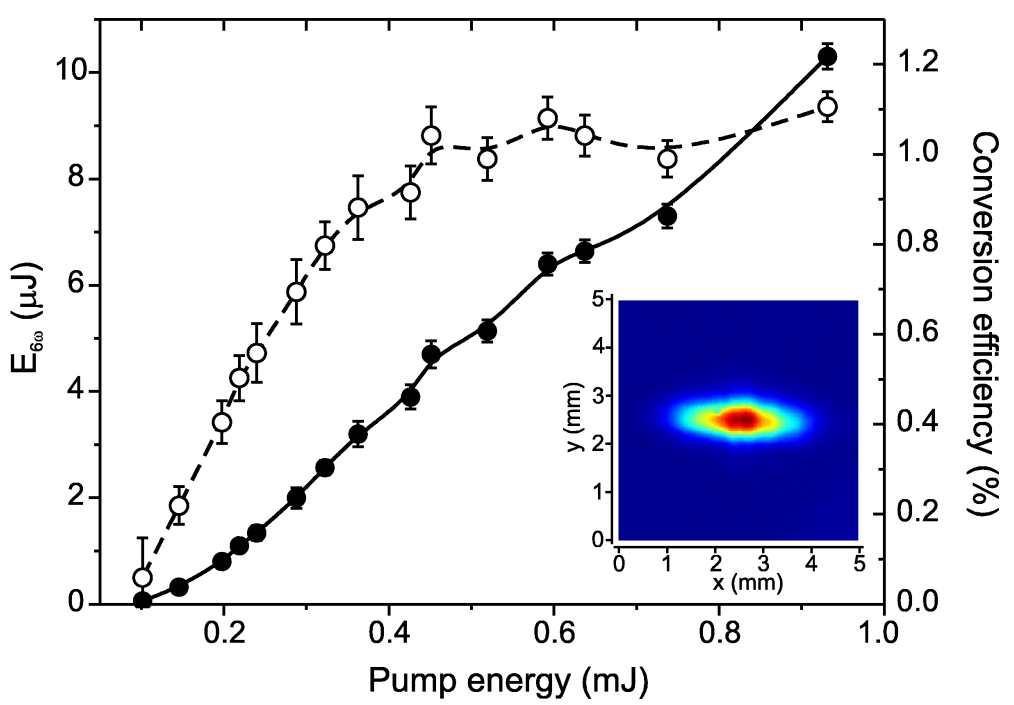

Fig. 9. Sixth-harmonic pulse energy $E_{6 \omega}$ (full circles, solid curve) and energy conversion efficiency (open circles, dashed curve) versus pump energy in 2-mm-long $\mathrm{MgF}_{2}$ crystal. Curves serve as guides for the eye. Inset shows the intermediate-field image of the intensity distribution of the sixth-harmonic beam.

ence $\mathrm{MgF}_{2}$ crystal shows no photo-induced darkening (colour centre formation).

And finally, we have estimated the sixth-harmonic pulsewidth considering that the duration of the sixthharmonic pulse equals to the time separation between the fastest (fourth-harmonic) and the slowest (sixthharmonic) pulses over the crystal length. The calculated projections of the group velocities are $v_{2 \omega}=$ $0.67925 c, v_{4 \omega}=0.68591 c$, and $v_{6 \omega}=0.61191 c$ along the propagation axis $(z)$ direction, as illustrated in Fig. 8(b), taking account of noncollinear propagation and pulse-front tilt of the second harmonic pulse. A simple estimation yields $\tau_{6 \omega}=l\left(1 / v_{6 \omega}-1 / v_{4 \omega}\right) \approx$ $1.2 \mathrm{ps}$, provided that the two-photon absorption for the sixth-harmonic radiation is small.

\section{Conclusions and outlook}

In conclusion, we have demonstrated efficient frequency conversion of 1-ps laser pulses in the ultraviolet by means of phase-matched four-wave optical parametric amplification and four-wave difference-frequency generation in transparent bulk solids with Kerr nonlinearity. The key point in realizing efficient four-wave interactions in isotropic media relies on fulfilment of the noncollinear phase matching condition using cylindrical (one-dimensional) beam focusing geometry. In this configuration, interaction of high energy pulses can be attained without the onset of uncontrollable intense pump beam break-up leading to filamentation and optical damage of the nonlinear medium.
Under these experimental settings, FWOPA provides the amplified signal energy as high as $55 \mu \mathrm{J}$ with almost $4 \%$ pump-to-signal energy conversion efficiency for the signal wavelengths around $330 \mathrm{~nm}$ in bulk fused silica and $\mathrm{CaF}_{2}$ crystals with 1-mJ pump energy. Moreover, FWOPA is able to support a broad amplification bandwidth ( $\sim 20 \mathrm{~nm}$ FWHM), as demonstrated experimentally and verified by calculations in the framework of the developed theoretical model. In particular, our calculations reveal that the two-fold parametric gain bandwidth broadening is resulting from self- and cross-phase-modulation effects imposed by the intense pump wave, whose contribution increases with increasing pump intensity. This result could be of particular practical interest since it demonstrates a potential to amplify sub-10 fs ultraviolet light pulses in a bulk solid state medium.

We also demonstrated the feasibility of the fourwave difference-frequency mixing technique in UVtransparent solids for generation of the ultraviolet harmonics of the Nd:glass laser. Specifically, efficient generation of third-harmonics at $351 \mathrm{~nm}$ in fused silica (with $15 \%$ energy conversion efficiency) and fifthharmonics at $211 \mathrm{~nm}$ in $\mathrm{CaF}_{2}$ and $\mathrm{MgF}_{2}$ crystals (with $10 \%$ energy conversion efficiency) was experimentally demonstrated. We also have shown that the pulse/beam physical overlap area and therefore the overall efficiency of the four-wave difference-frequency mixing process in the noncollinear interaction at large phase matching angles is greatly improved by pulse-front tilting. Complementary measurement of the nonlinear transmission at $264 \mathrm{~nm}$ (i. e. wavelength of the shortest 
pump pulse) has yielded two-photon absorption coefficients of $\mathrm{CaF}_{2}$ and $\mathrm{MgF}_{2}$, indicating that the latter crystal possess the lowest two-photon absorption $\left(\beta_{264}=1.9 \cdot 10^{-12} \mathrm{~cm} / \mathrm{W}\right)$ among the UV-transparent solid-state materials. Taking this advantage, we have demonstrated for the first time to our knowledge an efficient generation of VUV radiation by means of fourwave difference-frequency mixing in $\mathrm{MgF}_{2}$, obtaining ultrashort light pulses with energy as high as $\sim 10 \mu \mathrm{J}$ at $176 \mathrm{~nm}$ (e. g. sixth-harmonic of the Nd:glass laser) with high beam quality and reasonably high energy conversion efficiency $(>1 \%)$.

The four-wave difference-frequency mixing approach in transparent bulk solid state media could be easily extended and implemented for generation of tunable ultrashort light pulses in the ultraviolet and vacuum ultraviolet in particular, where conventional frequency conversion techniques in crystals with quadratic nonlinearity suffer from high linear and nonlinear absorption and severe phase matching constraints.

\section{Acknowledgement}

Authors acknowledge the financial support from the Lithuanian State Science and Studies Foundation (project FORTAS, No. B-29/2008).

\section{References}

[1] I.V. Hertel and V. Radloff, Ultrafast dynamics in isolated molecules and molecular clusters, Rep. Prog. Phys. 69, 1897-2003 (2006).

[2] J.H. Klein-Wiele, J. Bekesi, and P. Simon, Sub-micron patterning of solid materials with ultraviolet femtosecond pulses, Appl. Phys. A 79, 775-778 (2004).

[3] I. Zergioti, A. Karaiskou, D.G. Papazoglou, C. Fotakis, M. Kaspetaki, and D. Kefetzopoulos, Femtosecond laser microprinting of biomaterials, Appl. Phys. Lett. 86, 163902 (2005).

[4] A.I. Kalachev, D.N. Nikogosyan, and G. Brambilla, Long-period fiber grating fabrication by high-intensity femtosecond pulses at $211 \mathrm{~nm}$, J. Lightwave Technol. 23, 2568-2578 (2005).

[5] I. Zergioti, K.D. Kyrkis, D.G. Papazoglou, and S. Tzortzakis, Structural modifications in fused silica induced by ultraviolet fs laser filaments, Appl. Surf. Sci. 253, 7865-7868 (2007).

[6] V. Zorba, N. Boukos, I. Zergioti, and C. Fotakis, Ultraviolet femtosecond, picosecond and nanosecond laser microstructuring of silicon: structural and optical properties, Appl. Opt. 47, 1846-1850 (2008).
[7] S. Backus, J. Peatross, Z. Zeek, A. Rundquist, G. Taft, M.M. Murnane, and H.C. Kapteyn, 16-fs, $1-\mu \mathrm{J}$ ultraviolet pulses generated by third-harmonic conversion in air, Opt. Lett. 21, 665-667 (1996).

[8] C.G. Durfee III, S. Backus, M.M. Murnane, and H.C. Kapteyn, Ultrabroadband phase-matched optical parametric generation in the ultraviolet by use of guided waves, Opt. Lett. 22, 1565-1567 (1997).

[9] C.G. Durfee III, S. Backus, H.C. Kapteyn, and M.M. Murnane, Intense 8-fs pulse generation in the deep ultraviolet, Opt. Lett. 24, 697-699 (1999).

[10] L. Misoguti, S. Backus, C.G. Durfee, R. Bartels, M.M. Murnane, and H.C. Kapteyn, Generation of broadband VUV light using third-order cascaded processes, Phys. Rev. Lett. 87, 013601 (2001).

[11] A.E. Jailaubekov and S.E. Bradforth, Tunable 30femtosecond pulses across the deep ultraviolet, Appl. Phys. Lett. 87, 021107 (2005).

[12] T. Fuji, T. Horyo, and T. Suzuki, Generation of $12 \mathrm{fs}$ deep-ultraviolet pulses by four-wave mixing through filamentation in neon gas, Opt. Lett. 32, 2481-2483 (2007).

[13] K. Kosma, S.A. Trushin, W.E. Schmid, and W. Fuss, Vacuum ultraviolet pulses of $11 \mathrm{fs}$ from fifth-harmonic generation of a Ti:sapphire laser, Opt. Lett. 33, 723725 (2008).

[14] L. Bergé and S. Skupin, Sub-2 fs pulses generated by self-channeling in the deep ultraviolet, Opt. Lett. 33, 750-752 (2008).

[15] U. Graf, M. Fiess, M. Schultze, R. Kienberger, F. Krausz, and E. Goulielmakis, Intense few-cycle light pulses in the deep ultraviolet, Opt. Express 16, 1895618963 (2008).

[16] H. Okamoto and M. Tatsumi, Generation of ultrashort light pulses in the mid-infrared $\left(3000-800 \mathrm{~cm}^{-1}\right)$ by four-wave mixing, Opt. Commun. 121, 63-68 (1995).

[17] H. Crespo, J.T. Mendonça, and A. Dos Santos, Cascaded highly nondegenerate four-wave-mixing phenomenon in transparent isotropic condensed media, Opt. Lett. 25, 829-831 (2000).

[18] H.-K. Nienhuys, P.C.M. Planken, R.A. van Santen, and H.J. Bakker, Generation of mid-infrared pulses by $\chi^{(3)}$ difference frequency generation in $\mathrm{CaF}_{2}$ and $\mathrm{BaF}_{2}$, Opt. Lett. 26, 1350-1352 (2001).

[19] R.V. Volkov, D.V. Khakhulin, and A.B. Savel'ev, Fourwave parametric conversion of femtosecond laser pulse in a filament induced in a solid target, Opt. Lett. 33, 666-668 (2008).

[20] J. Liu and T. Kobayashi, Cascaded four-wave mixing and multicolored arrays generation in a sapphire plate by using two crossing beams of femtosecond laser, Opt. Express 16, 22119-22125 (2008).

[21] P.S. Banks, M.D. Feit, and M.D. Perry, Highintensity third-harmonic generation in beta barium borate through second-order and third-order susceptibilities, Opt. Lett. 24, 4-6 (1999). 
[22] J.-P. Fève, B. Boulanger, and Y. Guillien, Efficient energy conversion for cubic third-harmonic generation that is phase matched in $\mathrm{KTiOPO}_{4}$, Opt. Lett. 25, 1373-1375 (2000).

[23] T. Schneider, R.P. Schmid, and J. Reif, Efficient self phase matched third harmonic generation of ultrashort pulses in a material with positive dispersion, Appl. Phys. B 72, 563-565 (2001).

[24] A. Penzkofer, J. Kraus, and J. Sperka, Noncollinear phase matched four photon frequency mixing in water, Opt. Commun. 37, 437-441 (1981).

[25] G. Mao, Y. Wu, and K.D. Singer, Third harmonic generation in self-focused filaments in liquids, Opt. Express 15, 4857-4862 (2007).

[26] A. Dubietis, G. Tamošauskas, P. Polesana, G. Valiulis, H. Valtna, D. Faccio, P. Di Trapani, and A. Piskarskas, Highly efficient four-wave parametric amplification in transparent bulk Kerr medium, Opt. Express 15, 11126-11132 (2007).

[27] H. Valtna, A. Dubietis, G. Tamošauskas, P. Polesana, J. Galinis, D. Majus, G. Valiulis, D. Faccio, P. Di Trapani, and A. Piskarskas, Efficient four-wave parametric amplification and spatial soliton generation in transparent isotropic medium with Kerr nonlinearity, Lithuanian J. Phys. 47, 403-410 (2007).

[28] H. Valtna, A. Dubietis, G. Tamošauskas, and A. Piskarskas, High-energy broadband four-wave optical parametric amplification in bulk fused silica, Opt. Lett. 33, 971-973 (2008).

[29] J. Darginavičius, G. Tamošauskas, G. Valiulis, and A. Dubietis, Broadband four-wave optical parametric amplification in bulk isotropic media in the ultraviolet, Opt. Commun. 282, 2995-2999 (2009).

[30] J. Darginavičius, D. Majus, G. Tamošauskas, and A. Dubietis, Highly efficient third harmonic generation by means of four-wave difference-frequency mixing in fused silica, Lithuanian J. Phys. 49, 171-174 (2009).

[31] A. Penzkofer and H.J. Lehmeier, Theoretical investigation of noncollinear phase-matched parametric fourphoton amplification of ultrashort light pulses in isotropic media, Opt. Quantum Electron. 25, 815-844 (1993).

[32] B.C. Stuart, M.D. Feit, S. Herman, A.M. Rubenchik, B.W. Shore, and M.D. Perry, Nanosecond-tofemtosecond laser-induced breakdown in dielectrics, Phys. Rev. B 53, 1749-1761 (1996).

[33] A. Dubietis, G. Tamošauskas, A. Varanavičius, and G. Valiulis, Two-photon absorbing properties of ultraviolet phase-matchable crystals at 264 and $211 \mathrm{~nm}$, Appl. Opt. 39, 2437-2440 (2000).

[34] D.N. Nikogosyan, Properties of Optical and LaserRelated Materials (John Wiley \& Sons, Chichester, 1997).

[35] Handbook of Optics, eds. M. Bass, E.W. Van Stryland, D.R. Williams, and W.L. Wolfe, 2nd ed. (McGraw-Hill Professional, New York, 1994).

[36] F. Seifert, J. Ringling, F. Noack, V. Petrov, and O. Kittelmann, Generation of tunable femtosecond pulses to as low as $172.7 \mathrm{~nm}$ by sum-frequency mixing in lithium triborate, Opt. Lett. 19, 1538-1540 (1994).

[37] V. Petrov, F. Noack, F. Rotermund, M. Tanaka, and Y. Okada, Sum-frequency generation of femtosecond pulses in $\mathrm{CsLiB}_{6} \mathrm{O}_{10}$ down to $175 \mathrm{~nm}$, Appl. Opt. 39, 5076-5079 (2000).

[38] V. Petrov, F. Rotermund, and F. Noack, Generation of femtosecond pulses down to $166 \mathrm{~nm}$ by sum-frequency mixing in $\mathrm{KB}_{5} \mathrm{O}_{8} \cdot 4 \mathrm{H}_{2} \mathrm{O}$, Electron. Lett. 34, 17481750 (1998).

\title{
ULTRATRUMPŲJU UV IMPULSU GENERAVIMAS IR STIPRINIMAS KETURBANGE PARAMETRINE SĄVEIKA SKAIDRIOSE KIETAKŪNĖSE TERPĖSE
}

\author{
A. Dubietis, J. Darginavičius, G. Tamošauskas, G. Valiulis, A. Piskarskas
}

Vilniaus universitetas, Vilnius, Lietuva

\begin{abstract}
Santrauka
Ištirtas efektyvus ultratrumpujų ultravioletinių šviesos impulsu generavimas, naudojant faziškai sinchroninį keturbangi parametrini šviesos stiprinimą ir keturbangị skirtuminio dažnio maišymą skaidriose kieto kūno terpèse su kubiniu netiesiškumu. Teoriškai ištirtas ir eksperimentiškai pademonstruotas plataus spektro impulsu parametrinis stiprinimas ties $330 \mathrm{~nm}$ bangos ilgiu izotropinėse terpèse lydyto kvarco ir $\mathrm{CaF}_{2}$ kristaluose. Pasiekti rezultatai leidžia tikètis,
\end{abstract}

kad tokiu metodu gali būti stiprinami itin mažos trukmės (7,5 fs) ultravioletiniai šviesos impulsai, kaupinant 1 ps trukmès regimosios šviesos impulsais su milidžauline energija. Taikant keturbangi skirtuminio dažnio generavimo metodą, eksperimentiškai gautas efektyvus Nd:stiklo lazerio trečiosios $(351 \mathrm{~nm})$, penktosios $(211 \mathrm{~nm})$ ir šeštosios $(176 \mathrm{~nm})$ harmonikų žadinimas lydyto kvarco, $\mathrm{CaF}_{2}$ ir $\mathrm{MgF}_{2}$ kristaluose. 